\title{
Estimating the Effect of Mobile Carriers on Time Synchronization by Point Interpolation
}

\author{
Zihao Chen ${ }^{1,2}$, Liangfu Peng ${ }^{* 1,2}$, Yifan Wang ${ }^{1}$ \\ ${ }^{1}$ College of Electrical \& Information Engineering, Southwest Minzu University, Chengdu 610041, China \\ ${ }^{2}$ Guangxi Key Laboratory of Precision Navigation Technology and Application, Guilin University of Electronic \\ Technology, Guilin 541004, China \\ Email: $1275153536 @ q q . c o m$
}

\begin{abstract}
During the bidirectional time signal transmission, the position of the motion carrier will produce a certain displacement relative to the starting position. When the displacement of the motion carrier has a great influence on the signal transmission time, in order to solve the high-precision time synchronization problem between the downloading bodies of arbitrary motion trajectories, a geometric model for the position change of the carrier is constructed. The position coordinates of the sampling points are obtained by satellite positioning, and the motion trajectory equation of the carrier is constructed by interpolation estimation. By interpolating the complex motion trajectory of the carrier and accurately analyzing the velocity vector of the motion carrier according to the trajectory, the clock difference between the two motion carriers is obtained. Computer simulation results show that for any motion carrier, the difference of sampling points will cause the time synchronization result to change within a certain range.
\end{abstract}

Keywords: Time synchronization; satellite positioning; function interpolation; average speed

\section{Introduction}

The precise timing of GNSS is increasingly important for the impact of ground carrier (Mainly carrying a large number of electronic equipment) time synchronization. However, in many cases, due to the influence of atmospheric and equipment factors, the transmission of satellite timing signals will be disturbed, and the ground carriers need to transmit time synchronization signals to each other. Military equipment such as ships, aircraft, and tracking missiles require high time synchronization accuracy, and it is necessary to consider that the transmission time of the time signal is affected by the carrier displacement during the motion. For example, the irregular motion caused by the waves or the carrier undergoes multiple lane changes to make a complex change in the motion trajectory. In order to reduce the time synchronization error due to carrier motion, it is necessary to consider the influence of the motion of the carrier.

The satellite locates the spatial coordinates. Even if the ground carrier cannot receive the satellite timing signal in time, the coordinates of the satellite positioning carrier can be used to calculate the clock difference by using the two-way transmission method, and then the propagation delay generated by the carrier motion is calculated. Therefore, it is not necessary for the satellite to transmit the timing signal at all times. At present, the time synchronization for motion vectors only gives some algorithms for the precise ranging of inter-satellite links, mainly including STDD and PTDD two model algorithms [1]. However, the calculation of the orbit of the inter-satellite link does not solve the motion of the ground carrier under complex orbits. The STDD algorithm is mainly applied to the case where the satellite's radial velocity changes are small and the trajectory changes are negligible. In the actual problem, the velocity vector changes greatly, and the STDD algorithm needs to obtain the average radial velocity of the complex motion before it can be applied. The algorithm will be compared later in this paper. In order to solve the above problems, this paper proposes a motion carrier time synchronization point interpolation estimation algorithm based on its original physical model. This algorithm can simulate the ground carrier motion trajectory by satellite positioning, accurately calculate the radial velocity, and can get the influence of velocity vector variation. It can also be solved by using the positioning point after acquiring the coordinates. Even in the face of very complex, very fast moving carrier algorithms are still valid. 


\section{Time Synchronization Algorithm Model}

\subsection{Time Signal Bidirectional Transfer Model of Motion Carrier [1]}

The two-way transmission time signal model is as follows: 1) The two carriers in motion respectively transmit a signal carrying a local time stamp to the other party during the movement. A first sends out the local clock to B, after receiving the local clock of the carrier A, the carrier B transmits the local clock of $\mathrm{B}$ to $\mathrm{A}$, after receiving the data, A records the time of T1, T2, and T3; 2) After receiving the local clock sent by the other party, the signal is captured, demodulated, the information frame is restored, and the time is calculated. 3) The clock difference can be determined by the local clock signal and the signal modulation and demodulation time transmitted between the carriers; 4) Establish geometric models for function construction and simulation of motion trajectories.

The parameters defined in Figure 1 are as follows [1]: Carrier A, Carrier B. $V_{A}$ is the velocity vector of the carrier $\mathrm{A}, V_{B}$ is the velocity vector of the carrier $\mathrm{B}$. T1 is the moment that A passes local clock information to $\mathrm{B}$, and $\mathrm{T} 2$ is the moment that $\mathrm{B}$ passes local clock information to $\mathrm{A}$. $t_{1}$ is the propagation delay of the signal from $\mathrm{A}$ to $\mathrm{B}$, and $t_{2}$ is the propagation delay of the signal from $\mathrm{B}$ to $\mathrm{A}$. $\Delta t$ is the clock difference between the two carriers $\mathrm{A}$ and $\mathrm{B}, \gamma_{1}$ and $\gamma_{2}$ are the uncertainties (which can be defined as constants) that cause errors. The initial distance between the two carriers A and B is $a$, and the distance between the two carriers when the $\mathrm{B}$ receives the signal transmitted from $\mathrm{A}$ is $b . \theta$ and $\varphi, c$ is the speed of light. Dotted line is a random motion path.

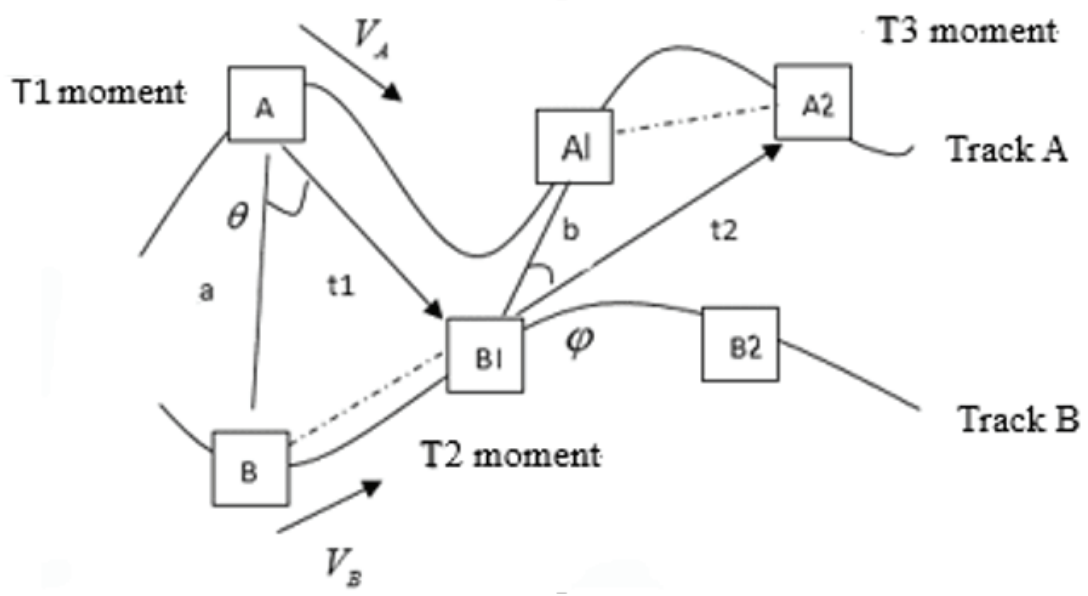

Figure 1. Physical model of two-way time signal transmission.

The expression of the clock difference $\Delta t$ can be derived from the physical model [8] transmitted by the bidirectional time signal:

$$
\left\{\begin{array}{l}
T 2=T 1+\Delta t+c t_{1}+\gamma_{2} \\
T 3=T 2+c t_{2}-\Delta t+\gamma_{1}
\end{array}\right.
$$

The expression of the clock difference $\Delta t$ can be obtained from (1):

$$
\Delta t=T 2+\frac{\left(c t_{2}-c t_{1}\right)}{2}-\frac{(T 1+T 3)}{2}-\frac{\left(\gamma_{1}+\gamma_{2}\right)}{2}
$$

Taking the triangle ABB1 as an example, according to the cosine theorem of the geometric relationship of triangles, the following can be obtained[2]:

$$
\cos \theta=\frac{a^{2}+\left(c t_{1}\right)^{2}-\left(V_{B} t_{1}\right)^{2}}{2 a c t_{1}}
$$

The same reason triangle A1B1A2 can be obtained:

$$
\cos \varphi=\frac{b^{2}+\left(c t_{2}\right)^{2}-\left(V_{A} t_{2}\right)^{2}}{2 b c t_{2}}
$$


Transform formula (3) to get:

$$
\left(c-V_{B}\right)^{2} t_{1}^{2}-2 a c \cos \theta t_{1}+a^{2}=0
$$

Similarly, the formula (4) can be transformed:

$$
\left(c-V_{A}\right)^{2} t_{2}^{2}-2 b c \cos \varphi t_{2}+b^{2}=0
$$

$V_{A}$ and $V_{B}$ are lateral velocity scalars.

\subsection{Interpolation Method for Fitting Motion Trajectories}

The randomness of the trajectory during the two-way time synchronization causes the carrier motion velocity to have a large error. Moreover, in the process of transmitting and receiving signals, the propagation delays of $\mathrm{A}$ and $\mathrm{B}$ are unknown, and it is impossible to calculate the random motion of the carrier to cause time synchronization error. Therefore, the satellite positioning method is used to obtain the position coordinates of the carrier motion to a certain moment [3].

Establish a Cartesian coordinate system with the center of the earth as the center. Taking the two-dimensional coordinates as an example, the coordinate conversion formula is as follows:

$$
\begin{aligned}
& x=R \cos (\alpha) \cos (\beta) \\
& y=R \cos (\alpha) \sin (\beta)
\end{aligned}
$$

$R$ is the radius of the Earth, $\alpha, \beta$ are latitude and longitude, respectively. Assume that the coordinates of the latitude and longitude conversion of satellite positioning are $\left(x_{1}, y_{1}\right),\left(x_{2}, y_{2}\right),\left(x_{3}, y_{3}\right), \ldots \ldots,\left(x_{n+1}, y_{n+1}\right)$. Sampling of the sampling points is as close as possible to the inflection point of the propagation delay, and at least one sampling point is maintained for each steering. This will make the data fit more precise. Construct x0y using the parallel lines of the line connecting the initial point and the last point.

Since the satellite positioning points are discontinuous, a function is needed to fit the trajectory of the carrier. Assume that there is at least one sampling point at each corner. Missing sampling points will lead to excessive trajectory prediction error. Although the sampling point is too much, the accuracy is improved, but the sampling data set is huge, the information processing speed is affected, and the over-fitting function is calculated more. It is complicated and time consuming.

For the $n+1$ samples $\left(x_{1}, y_{1}\right) \sim\left(x_{n+1}, y_{n+1}\right)$, the interpolation function method is used to calculate the carrier trajectory equation:

1) For trajectory sampling points with fewer constraints, a polynomial is used. Since the interpolation condition contains $n+1$ constraints, and the $n$-degree polynomial contains $n+1$ undetermined coefficients, the non-singular matrix (Vandermonde matrix) composed of mutually different interpolation nodes has a unique solution. The interpolation equation can be obtained by taking $n+1$ constraints.

2) For more complex motions and $n+1$ nodes with too many constraints, Lagrange interpolation is generally used to avoid the difficulty of solving higher-order functions of equations.

Substituting the sampling points of the $n+1$ target trajectories acquired by the satellite into the interpolation basis function can obtain a set of function bases. $i \in(0, n)$. Substituting each sample point coordinate into a Lagrangian interpolation polynomial:

$$
L_{i}(x)=\sum_{i=0}^{n} f\left(x_{i}\right) l_{i}(x)
$$

The function model is shown in Figure. 2:

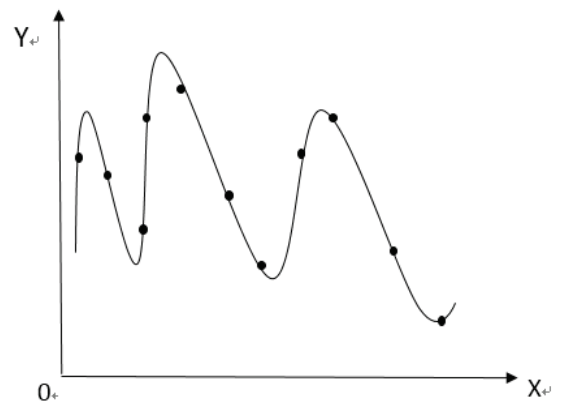


Figure 2. Trajectory model of carrier B moving to T2

\section{Time Delay Caused by Motion}

\subsection{Lateral Speed during Propagation Time}

Assume that most of the continuous trajectory equations represented by the $n$th degree polynomial after the interpolation operation are as follows:

$$
f(x)=a_{0}+a_{1} x+a_{2} x^{2}+a_{3} x^{3}+\cdots+a_{n} x^{n}
$$

A differential operation is performed on the trajectory function to obtain a velocity vector at any time.

$$
f^{\prime}(x)=a_{1}+2 a_{2} x+3 a_{3} x^{2}+\cdots+n a_{n} x^{n-1}=\sum_{i=1}^{n} i a_{i} x^{i-1}
$$

Substituting $\left(x_{B 1}, y_{B 1}\right) \sim\left(x_{B n+1}, y_{B n+1}\right)$ can get the speed of the carrier B at any time. The lateral velocity of the carrier $\mathrm{B}$ is decomposed using the Pythagorean theorem as follows:

$$
V_{B}^{2}=\lambda^{2}+\left(\left|k_{B}\right| \lambda\right)^{2}
$$

$k_{B}$ is the tan value of the vector decomposition of the arbitrary point velocity, and $\lambda$ is the lateral velocity scalar after decomposition. According to formula (10):

$$
\lambda=\frac{V_{B}}{\sqrt{\left|k_{B}\right|^{2}+1}}
$$

The trajectory function is integrated over time $t_{1}$ to obtain the lateral mean of the velocity, expressed as $\bar{V}_{B t}$ :

$$
\bar{V}_{B t}=\frac{\int_{\Delta x} \frac{V_{B}}{\sqrt{\left|\sum_{i=1}^{n} i a_{i} x^{i-1}\right|^{2}+1}} d x}{\Delta x}
$$

In the formula, $\alpha_{i}$ is a polynomial coefficient. Similarly, the lateral mean $\bar{V}_{A t}$ of the velocity can be obtained by integrating in time $t_{2}$.

\subsection{Calculate the Propagation Time t1, t2}

$a$ is the initial distance of $\mathrm{A}, \mathrm{B}$, according to the distance formula between two points:

$$
a=\sqrt{\left(x_{B 1}-x_{A 1}\right)^{2}+\left(y_{B 1}-y_{A 1}\right)^{2}}
$$

The range of values of $\cos \theta$ is determined by the angle between $\left(x_{B 1}, y_{B 1}\right) \sim\left(x_{B n+1}, y_{B n+1}\right)$. According to the initial coordinate $\left(x_{A 1}, x_{B 1}\right)$ of point $\mathrm{A}$, the two-point distance formula is made with $\left(x_{B 1}, y_{B 1}\right),\left(x_{B n+1}, y_{B n+1}\right)$. The expression of $\cos \theta$ is obtained by the cosine theorem [4]:

$$
\cos \theta=\frac{\left(x_{B 1}-x_{A 1}\right)^{2}+\left(y_{B 1}-y_{A 1}\right)^{2}+\left(x_{B n+1}-x_{A 1}\right)^{2}+\left(y_{B n+1}-y_{A 1}\right)^{2}-\left(x_{B 1}-x_{B n+1}\right)^{2}+\left(y_{B 1}-y_{B n+1}\right)^{2}}{2 \sqrt{\left[\left(x_{B 1}-x_{A 1}\right)^{2}+\left(y_{B 1}-y_{A 1}\right)^{2}\right]\left[\left(x_{B n+1}-x_{A 1}\right)^{2}+\left(y_{B n+1}-y_{A 1}\right)^{2}\right]}}
$$

Substituting $\cos \theta$ into carrier A for carrier B propagation delay formula (5) can be obtained:

$$
\left(c-\bar{V}_{B t}\right)^{2} t_{1}^{2}-2 a c \cos \theta t+a_{1}^{2}=0
$$

Using the quadratic function to solve the value of $t_{1}$ :

$$
t_{1}=\frac{2 a c \cos \theta+\sqrt{(2 a c \cos \theta)^{2}-4 a^{2}\left(c-\bar{V}_{B t}\right)^{2}}}{2\left(c-\bar{V}_{B t}\right)^{2}}
$$

Similarly, the value of $t_{2}$ can be obtained by substituting $\cos \varphi$ into carrier B for carrier A propagation delay formula (6): 


$$
t_{2}=\frac{2 b c \cos \varphi+\sqrt{(2 b c \cos \varphi)^{2}-4 b^{2}\left(c-\bar{V}_{A t}\right)^{2}}}{2\left(c-\bar{V}_{A t}\right)^{2}}
$$

Let the difference between the propagation delays be $\Delta T=t_{2}-t_{1}$, and the relational expression can be substituted into the formula (2) to obtain the clock difference $\Delta t$ :

$$
\Delta t=T 2-\frac{(T 1+T 3)-\left(\gamma_{1}+\gamma_{2}\right)}{2}+\frac{c}{2} \Delta T
$$

\section{Simulation and Analysis}

Assume that the motion trajectory of the carrier has been obtained after positioning, and satellite positioning constructs a trajectory with 10 points. Using a computer to simulate satellites randomly taking points on a prescribed path. The different positioning points of each group determine that the trajectory equation is also different. The simulation is performed based on the five trajectory samples of the motion trajectory configurations A and B. Figure 3 shows the trajectory equation of carrier A in A1 to A2 time, and Figure 4 shows the trajectory equation of carrier B in B and B1 time.

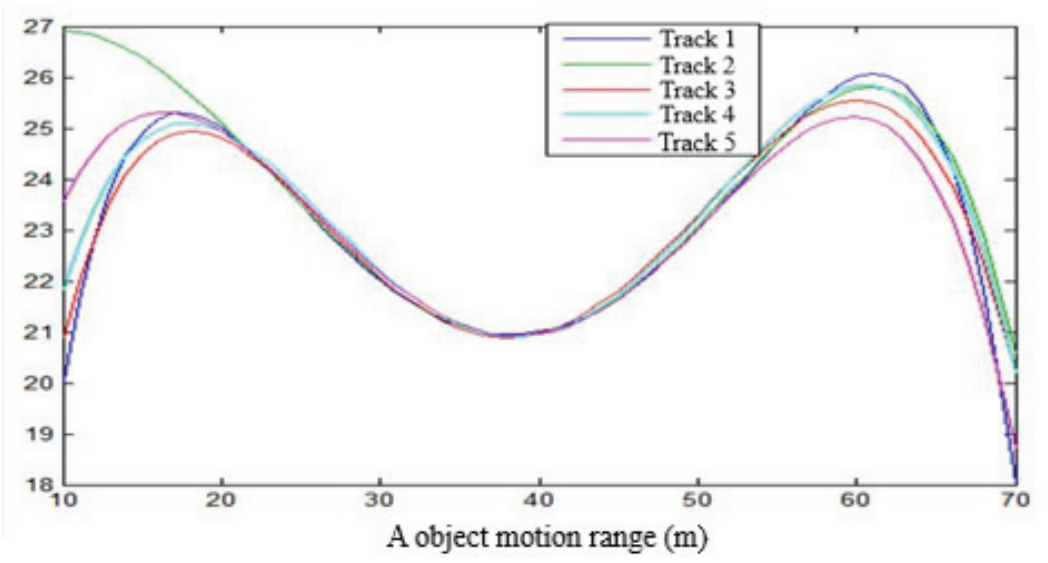

Figure 3. Location acquired trajectory of A carrier

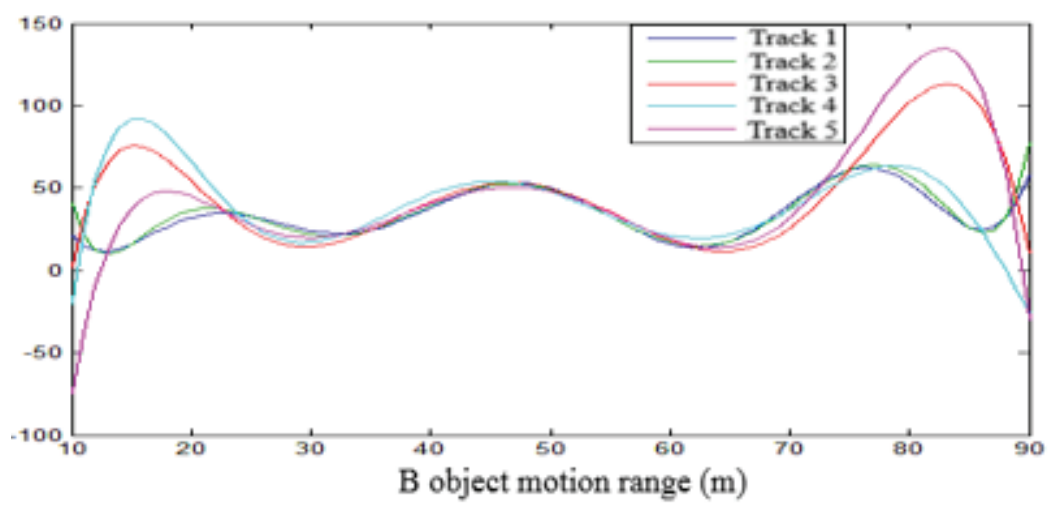

Figure 4. Location acquired trajectory of B carrier

It is assumed that the trajectory 1 of the A carrier and the trajectory 1 of the B carrier are calculated, the trajectory equations for $y_{A}, y_{B}$ are calculated by sampling point interpolation as follows:

$$
y_{A}=P_{A} \times W_{A}, y_{B}=P_{B} \times W_{B}
$$

The matrix $P_{A}$ and $P_{B}$ are polynomial coefficients. 


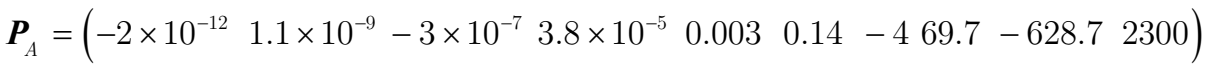

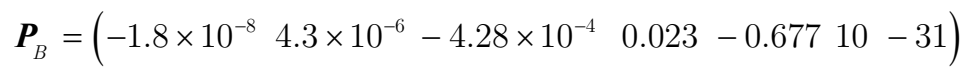

$$
\begin{aligned}
& \boldsymbol{W}_{A}=\left(\begin{array}{c}
x^{9} \\
x^{8} \\
\vdots \\
1
\end{array}\right), \boldsymbol{W}_{B}=\left(\begin{array}{c}
x^{6} \\
x^{5} \\
\vdots \\
1
\end{array}\right)
\end{aligned}
$$

Suppose that the motion threshold of the A carrier in the signal propagation time is in the range of 0 to $80 \mathrm{~m}$, and the motion threshold of the B carrier in the signal propagation time is in the range of 0 to 100 $\mathrm{m}$, the velocity of the $A$ vector and the $B$ vector at $x=0$ was $80 \mathrm{~km} / \mathrm{h}$ and $100 \mathrm{~km} / \mathrm{h}$, respectively.

The original function of the simulation path belongs to the higher-order function, but the order of the function within the signal transmission delay range is low, and the Lagrangian interpolation can meet the requirement. If the path of the carrier within the transit time delay belongs to a higher order function, such as the path generated in Figure 4. At this time, segmentation low-order polynomial interpolation is used. It consists of piecewise linear interpolation and piecewise cubic Hermite interpolation. The use of low-order interpolation between each cell not only improves the accuracy at non-interpolation points, but also avoids the phenomenon of the Runge.

The fitted trajectory and the real trajectory are error-prone. The five equations obtained by interpolation of different positioning points also have some differences. The more satellite positioning points obtained, the smaller the error. Taking the B carrier as an example, the B carrier trajectory 1 is calculated by the formula (11), and the lateral velocity at any time is obtained as shown in Figure 5:

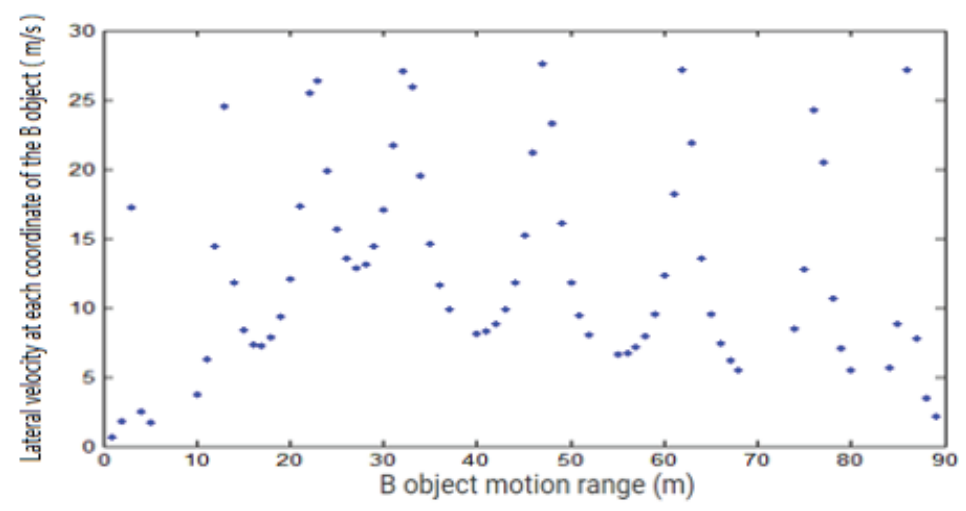

Figure 5. Lateral velocity of carrier B at any time

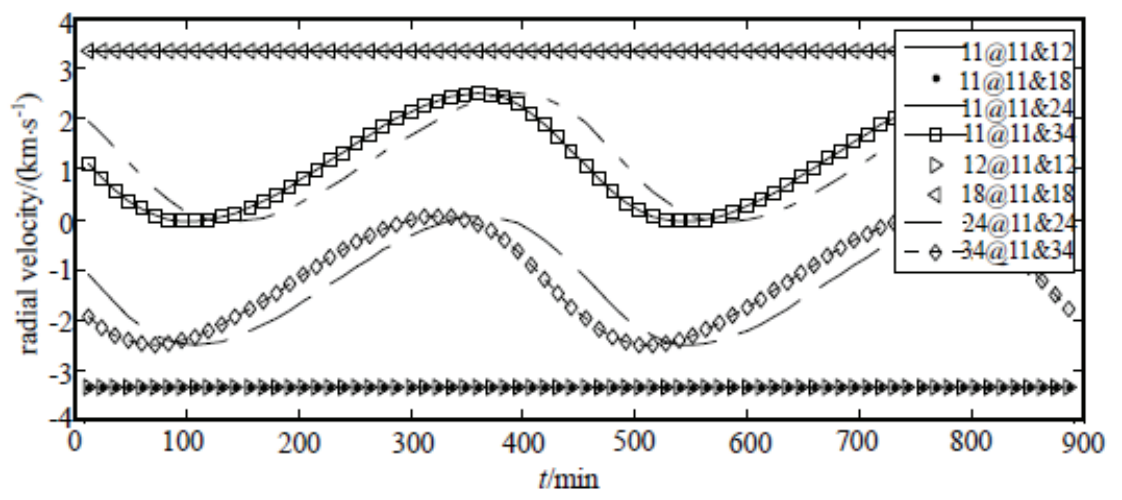

Figure 6. Radial velocity of carrier in STDD algorithms 
According to the results, it can be found that the degree of dispersion of the speed is large, and it also proves that the ISL algorithm STDD[1] cannot be directly applied to the time synchronization of the ground carrier. The effect of the clock difference caused by motion requires the integration of all speed values. After calculation, the speed of A and B carriers at track 1 is $\bar{V}_{A t}=13.355 \mathrm{~m} / \mathrm{s}, \bar{V}_{B t}=11.681 \mathrm{~m} / \mathrm{s}$. After placing the two results under the same reference frame and satisfying the value requirements, the initial coordinates of the motion signals transmitted by the motion carriers A and B are obtained using a given time reference. Calculate $\cos \theta$ and $\cos \varphi$ using coordinate values. Using equations (16) and (17) to obtain the effect of carrier motion on the clock difference $\Delta T=t_{2}-t_{1}$. It can be found from equations (16) and (17) that each of the fitted trajectories can obtain an average velocity, and the influence of the average carrier velocity on the propagation delay is mainly the value of $\sqrt{2 c \bar{V}} \mathrm{~m} / \mathrm{s}$. The influence on the synchronous clock difference $\Delta t$ is about 38 us.

\section{Conclusion}

The randomness of the motion trajectory of the carrier causes the velocity vector to change too much, so we need to create a new mathematical model without using an ISL algorithm. Since the error of the algorithm itself is caused by the change of the position and speed of the carrier, the modeling of the ground carrier by using the STDD algorithm directly is limited by the vector speed vector. In the complex model in which the STDD algorithm cannot be operated, only the known method can be obtained by using the point-taking method, so as to eliminate the influence of random motion and construct an equation for calculation.

The error term sources of the clock error $\Delta t$ obtained by combining the above formulas (16), (17), and (18) are mainly $\cos \theta$ and $\cos \varphi$. This means that the degree of dispersion of satellite positioning coordinates affects the establishment of the carrier motion trajectory equation and affects the average velocity. The coordinates of the initial point and the last point also have a greater influence on the accuracy of time synchronization. Although satellite positioning is required to make calculations cumbersome, it is possible to avoid the problem that the complex motion trajectory of the carrier cannot be calculated. The measurement accuracy of the carrier speed will be higher at any time, and the more the positioning points, the more accurate the calculation result of the motion of the carrier. Due to the complexity of the carrier's motion trajectory, different interpolation methods are sometimes required. For example, when the computational efficiency is low during computer operations, the Newtonian difference method can be used to make the calculation faster. In the process of using satellite positioning points, try to sample the motion carriers at equal intervals to ensure the fitting accuracy of the carrier motion trajectory.

\section{Acknowledgements.}

1. The work of this paper is supported by the Southwest Minzu University Graduate Innovative Research Project (Master Program CX2019SZ24).

2. The work of this paper is supported by Guangxi Key Laboratory of Precision Navigation Technology and Application, Guilin University of Electronic Technology (No.DH201911).

A special acknowledgement should give to Southwest Minzu University and Guilin University of Electronic Technology for its experimental conditions and technical support.

\section{References}

1. Xu Yong, Changqing, Yu Zhijian. Research on New Intersatellite Link Measurement and Communication Methods for GNSS [J]. School of Electronic Information Engineering, Beijing University of Aeronautics and Astronautics, 2011 (6): 234-235.

2. Mahong, Wang Yuanqin, Chen Gucang. Research on relative ranging and time synchronization between formation small satellites [J]. Journal of Equipment Command Technology College, 2006 (4): 67-71..

3. Wang Shufang, Wang Liliang. Time synchronization technology of satellite navigation and positioning system [J]. Global Positioning System, 2005, 30 (2): 10-14.

4. Zhang Lixin, Wang Wei, Wang Gang. Two-way comparison and long-distance time synchronization technology [J]. Space electronics technology, 2002 (2): 7-9. 
5. Zhong Xingwang, Chen Hao. Analysis and correction of the effect of satellite motion on inter-satellite bidirectional time synchronization [J]. China Space Science and Technology, 2007 (6): 54-58.

6. Holmes J K, Raghavan S. A summary of the new GPS IIR-M and IIF modernization signals. In: Proceedings of the 2004 IEEE Vehicular Technology Conference. Los Angeles: IEEE, 2004. 4116-4126

7. Zheng Jinjun, Lin Yiming, Chen Zhonggui, et al. Analysis of GPS inter-satellite link technology and autonomous navigation algorithm. Spacecraft Engineering, 2009, 18 (2): 28-35

8. Yu Xuehui, Wang Dun, Li Zhou, et al. Two-way comparison of high precision physical time synchronization method [J]. Journal of Aeronautics, 2019 (4): 1-15

9. Maine K P, Anderson P, Langer J. Crosslink for the next-generation GPS. In: Proceedings of the 2003 IEEE Aerospace Conference. IEEE,2003. 1-8

10. Feng Yanming. Research on theoretical problems of GPS precision positioning. Ph.D. thesis, Wuhan University of Surveying and Mapping Science and Technology. 1989:1-96.

11. Yan Ye, Yan Xiaoning, Ren Xuan. Autonomous positioning of satellite networks by inter-satellite ranging [J]. Journal of Space Science, 2000 (20): 54-60. 